\title{
Prenatal Cocaine Exposure and Adolescent Neural Responses to Appetitive and Stressful Stimuli
}

\author{
Sarah W Yip', Elise B Potenza ${ }^{2}$, Iris M Balodis', Cheryl M Lacadie ${ }^{3}$, Rajita Sinha ${ }^{1,2,4}$, Linda C Mayes ${ }^{2,5}$ and \\ Marc N Potenza*,1,2,4 \\ 'Department of Psychiatry, Yale University School of Medicine, New Haven, CT, USA; ${ }^{2}$ Child Study Center, Yale University School of Medicine, \\ New Haven, CT, USA; ${ }^{3}$ Department of Diagnostic Radiology, Yale University School of Medicine, New Haven, CT, USA; ${ }^{4}$ Department of \\ Neurobiology, Yale University School of Medicine, New Haven, CT, USA; ${ }^{5}$ Departments of Epidemiology, Pediatrics and Psychology, Yale \\ University School of Medicine, New Haven, CT, USA
}

Preclinical research has demonstrated the effects of prenatal cocaine exposure (PCE) on brain regions involved in emotional regulation, motivational control, and addiction vulnerability - eg, the ventral striatum (VS), anterior cingulate (ACC), and prefrontal cortex (PFC). However, little is known about the function of these regions in human adolescents with PCE. Twenty-two adolescents with PCE and 22 age-, gender-, and IQ-matched non-cocaine exposed (NCE) adolescents underwent functional magnetic resonance imaging ( $\mathrm{M} M \mathrm{RI}$ ) during exposure to individually personalized neutral/relaxing, stressful, and favorite-food cues. $\mathrm{fMRI}$ data were compared using grouplevel two-tailed t-tests in the Biolmage Suite. In comparison with NCE adolescents, PCE adolescents had reduced activity within cortical and subcortical brain regions, including the VS, ACC, and medial and dorslolateral PFC during exposure to favorite-food cues but did not differ in neural responses to stress cues. Subjective food craving was inversely related to dorsolateral PFC activation among PCE adolescents. Among PCE adolescents, subjective anxiety ratings correlated inversely with activations in the orbitofrontal cortex and brainstem during the stress condition and with ACC, dorsolateral PFC, and hippocampus activity during the neutral-relaxing condition. Thus adolescents with PCE display hypoactivation of brain regions involved in appetitive processing, with subjective intensities of craving and anxiety correlating inversely with extent of activation. These findings suggest possible mechanisms by which PCE might predispose to the development of addictions and related disorders, eg, substance-use disorders and binge-eating.

Neuropsychopharmacology (2014) 39, 2824-2834; doi:I0.I038/npp.20I4.I33; published online 16 July 2014

\section{INTRODUCTION}

Prenatal cocaine exposure (PCE) is associated with negative behaviors during early childhood, including externalizing and behavioral problems (Ackerman et al, 2010) and poor sustained attention (Ackerman et al, 2010), inhibitory control (Bridgett and Mayes, 2011) and working memory (Mayes et al, 2007). By contrast, effects of PCE during adolescence and young adulthood may be more subtle than those observed during early childhood (Liu and Lester, 2011) and are less well understood (Buckingham-Howes et al, 2013). Data suggest increased rates of substance use and obesity during middle childhood and adolescence following PCE (Delaney-Black et al, 2011; LaGasse et al, 2011; Rando et al, 2013; Richardson et al, 2013). Here, we use functional magnetic resonance imaging (fMRI) to investigate brain function underlying responses to stress and favorite-food cues in adolescents with and without PCE.

\footnotetext{
*Correspondence: Professor MN Potenza, Department of Psychiatry and Child Study Center, Yale University School of Medicine, Room S-104, 34 Park Street, New Haven, CT 06510, USA, Tel: + I 203 974 7356, Fax: + I 203974 7366, E-mail: marc.potenza@yale.edu Received 15 April 20।4; revised 15 May 20I4; accepted 21 May 2014; accepted article preview online 6 June 2014
}

Adolescence is a key period for the development of stress processing (Andersen and Teicher, 2008) and motivational control and is a vulnerable period for the development of addictions (Chambers and Potenza, 2003). Preclinical research has demonstrated significant effects of PCE on brain regions involved in emotional regulation, motivational control, and addiction vulnerability during adolescence (Chambers and Potenza, 2003)-eg, anterior cingulate (ACC) (Harvey, 2004), prefrontal cortex (PFC) (McCarthy and Bhide, 2012), and ventral striatum (VS) (Harvey et al, 2001; Wang et al, 2013). In humans, neurostructural (Rando et al, 2013) and neurofunctional (Li et al, 2009; Sheinkopf et al, 2009; Li et al, 2011) alterations (eg, during response inhibition, resting state alterations) in regions of the VS and PFC have also been reported among adolescents with PCE. Both stress and reward systems involving the VS, ACC, and PFC may contribute to engagement in risk behaviors, such as substance use (Sinha, 2009). However, no fMRI studies have examined the neural correlates of stress and craving among adolescents with and without PCE.

Neural responses to both stressful and appetitive cues are altered among obese (in comparison with lean) adults (Jastreboff et al, 2013), and increased rates of childhood 
obesity have been reported among children with PCE (LaGasse et al, 2011). However, the extent to which neural responses to stressful and appetitive stimuli may be altered among adolescents with PCE has not been assessed previously. Given that stress and appetitive processes may underlie engagement in eating and substance-use behaviors, the current study provides insight into how PCE may influence specific brain processes that could influence the risk for developing substance-use or weight-related problems.

We have previously demonstrated increased neural responses within reward-related regions among adults with cocaine dependence in response to stressful and appetitive (substance cue) stimuli (Potenza et al, 2012). In contrast, findings from adolescent studies suggest that decreased reward-related VS activity may be a risk-factor for the development of addictions (Peters et al, 2011). In addition, decreased activity within the PFC and ACC is associated with increased risk-taking among adolescents (Eshel et al, 2007). As such, we hypothesized that, in comparison with NCE adolescents, PCE adolescents would have altered activation within prefrontal-cortical and subcortical regions (in particular the ACC, PFC, and VS) during exposure to stressful and appetitive (favorite food) cues. Based on previous findings of a significant positive association between BOLD-signal responses within reward-related neurocircuitry and subjective ratings of stress and craving among adults with cocaine dependence (Potenza et al, 2012), we further hypothesized that neural activation within prefrontal-cortical and limbic regions would be positively correlated with self-reported ratings of anxiety and food craving among both PCE and NCE adolescents.

\section{METHODS}

\section{Participants and Screening}

Adolescents were recruited from a larger cohort of PCE and NCE children and adolescents who have been followed since birth (Mayes et al, 2005; Hommer et al, 2012; Rando et al, 2013). Maternal cocaine use was determined based on mother's self-report and on urine toxicology during pregnancy or immediately following delivery. The final sample included in this study consisted of 22 PCE adolescents (mean age $=14.9$ years $(\mathrm{SE}=0.25) ; 8$ female) and $22 \mathrm{NCE}$ adolescents (mean age $=14.5$ years $(\mathrm{SE}=0.13)$; 8 female) ranging between 14 and 17 years of age. Imaging data from 23 of these participants $(\mathrm{PCE}=13, \mathrm{NCE}=10)$ has been published previously (Hommer et al, 2012); however, this previous study did not compare adolescents' fMRI data based on PCE status. Current life stress was measured using the Perceived Stress Scale (PSS; Cohen et al, 1983) and the Negative Life Events Inventory (NLEI; Wills et al, 1992). IQ was assessed using the Kaufman Assessment Battery for Children (Kaufman, 1983). PCE and NCE adolescent groups did not differ in age $(\mathrm{F}=2.07, p=0.16)$, body mass index (BMI; $\mathrm{F}=0.17, p=0.69)$, IQ $(\mathrm{F}=3.73, p=0.06)$, PSS score $(\mathrm{F}=0.09, p=0.77)$, NLEI score, gender $\left(\chi^{2}=0.00, p=1.00\right)$, or race/ethnicity $\left(\chi^{2}=6.34, p=0.28\right)$. Complete demographic information is given in Table 1.

All participants were assessed using the National Institute of Health Diagnostic Interview Schedule for Children (C-DISC-4.0-Y) (Shaffer et al, 2000). None of the
Table I Demographic Characteristics of PCE and NCE

Adolescents

\begin{tabular}{|c|c|c|c|c|c|c|}
\hline & \multicolumn{2}{|c|}{$\operatorname{NCE}(n=22)$} & \multicolumn{2}{|c|}{ PCE $(n=22)$} & \multirow[t]{2}{*}{$\mathbf{F}$} & \multirow[t]{2}{*}{$P$} \\
\hline & Mean & SE & Mean & SE & & \\
\hline Age, years & 14.50 & 0.13 & $|4.9|$ & 0.25 & 2.07 & $>0.10$ \\
\hline BMI & 25.95 & 1.39 & 25.27 & 0.94 & 0.17 & $>0.10$ \\
\hline $\mathrm{IQ}^{\mathrm{a}}$ & 99.20 & 3.36 & 90.53 & 2.96 & 3.73 & $>0.05$ \\
\hline Negative life events ${ }^{b}$ & 4.45 & 0.59 & 5.09 & 0.76 & 0.44 & $>0.10$ \\
\hline \multirow[t]{2}{*}{ Perceived stress $^{c}$} & 11.18 & 0.73 & 11.50 & 0.79 & 0.09 & $>0.10$ \\
\hline & $\mathrm{N}$ & $\%$ & $n$ & $\%$ & $\chi^{2}$ & P \\
\hline \multicolumn{7}{|l|}{ Gender } \\
\hline Male & 14 & 63.6 & 14 & 63.6 & 0.00 & $>0.10$ \\
\hline Female & 8 & 36.4 & 8 & 36.4 & & \\
\hline \multicolumn{7}{|l|}{ Race/ethnicity $^{d}$} \\
\hline African American & 13 & 59.1 & 18 & 81.8 & 6.34 & $>0.10$ \\
\hline Caucasian & 3 & 13.6 & 2 & 9.1 & & \\
\hline Asian & 2 & 9.1 & । & 4.5 & & \\
\hline Hispanic & 0 & 0.0 & । & 4.5 & & \\
\hline Biracial & । & 4.5 & 0 & 0.0 & & \\
\hline \multicolumn{7}{|l|}{ Substance use (lifetime) } \\
\hline Alcohol & 6 & 27.3 & 11 & 50.0 & 2.40 & $>0.10$ \\
\hline Cigarettes & 5 & 22.7 & 9 & 40.9 & 1.68 & $>0.10$ \\
\hline Cannabis & 4 & 18.2 & 10 & 45.5 & 3.77 & $>0.05$ \\
\hline Steroids & 0 & 0.0 & 2 & 9.1 & 2.10 & $>0.10$ \\
\hline Opioids & 0 & 0.0 & 2 & 9.1 & 2.10 & $>0.10$ \\
\hline Cocaine & 0 & 0.0 & I & 4.5 & 1.02 & $>0.10$ \\
\hline Methamphetamine & 0 & 0.0 & । & 4.5 & 1.02 & $>0.10$ \\
\hline Inhalants & 0 & 0.0 & । & 4.5 & 1.02 & $>0.10$ \\
\hline
\end{tabular}

Abbreviations: BMI, body mass index; NCE, no prenatal cocaine exposure; PCE, prenatal cocaine exposure.

aMeasured using Kaufman Assessment Battery for Children; data missing for two NCE and three PCE participants.

${ }^{b}$ Negative Life Events Inventory score.

'Perceived Stress Scale score.

${ }^{\mathrm{d}}$ Race/ethnicity information missing for three NCE participants.

adolescents included in this study met criteria for any AxisI disorder, including substance-use disorders. Consistent with the estimates from the general population (SAMHSA, 2013), adolescents did report some substance use. Among these, alcohol use was most frequently reported $(n=17$, $38.6 \%)$, followed by tobacco $(n=14,31.81 \%)$, cannabis $(n=14,31.81 \%)$, steroids $(n=2,4.54 \%)$, opiates $(n=2$, $4.54 \%)$, cocaine $(n=1,2.27 \%)$, methamphetamine $(n=1$, $2.27 \%)$, and inhalants $(n=1,2.27 \%)$. PCE and NCE adolescent groups did not differ in lifetime use for any substance ( $p$ 's $>0.05$; Table 1). Further exclusion criteria for all participants included any contraindication to scanning.

\section{Individualized Imagery Method and Script Development}

Standardized, structured interviews were conducted using the Scene Development Questionnaire (Sinha, 2009), and the resulting data were used to create individually tailored neutral, stressful, and appetitive scripts as described 
previously (Sinha, 2009; Hommer et al, 2012; Jastreboff et al, 2013). This methodology is based on that originally developed by Lang et al (1980) and has since been used extensively in conjunction with fMRI scanning to study neural responses to stress, craving (in response to either food or drug cues), and neutral-relaxing cues; eg, (Sinha et al, 2005; Sinha, 2009; Jastreboff et al, 2011; Seo et al, 2011; Hommer et al, 2012; Potenza et al, 2012; Jastreboff et al, 2013; Seo et al, 2014). In this fMRI task, audiotaped personalized scripts based on individual interviews are presented de novo to the participant during scanning in order to induce either stress, craving or neutral-relaxed states (block design). The resulting fMRI data are analyzed in a block (rather than an event-related) manner. For a schematic overview of trial structure, see Supplementary Figure S1.

Stress scripts were based on participants' descriptions of life events during which they felt 'sad, mad or upset in that moment and could not do anything to change the situation' and were individually calibrated for level of stress across subjects based on participants' ratings $(0=$ 'not at all stressful'; $10=$ 'one of the most stressful experiences') so that only those events rated as an $\geqslant 8$ were included (Hommer et al, 2012). Appetitive scripts were based on participants' descriptions of recent times when they wanted and then consumed their favorite food (eg, pizza, ice cream) (Hommer et al, 2012) and were also individually calibrated so that only those events rated as an $\geqslant 8$ were included $(0=$ 'not at all pleasurable'; $10=$ 'one of the most pleasurable experiences'). Neutral-relaxing scripts were based on participants' descriptions of commonly experienced neutral or relaxing situations (eg, sitting in the park, relaxing in your room) (Hommer et al, 2012). For examples, see Supplementary Materials.

All scripts were standardized to ensure uniformity in the style, length, (approximately 250 to 400 words) and content format of the scripts across participants and conditions. Previous to scanning, each script was recorded on audiotape or digitally. All scripts were narrated by a female research using a neutral tone. Although there were no formal criteria for the length of pauses between words/ sentences, all scripts were narrated in a naturalistic manner without the addition of any extended pauses. In order to reduce possible individual variability in imagery ability, all participants conducted a relaxation and guided-imagery training session before scanning (Hommer et al, 2012).

\section{Functional Magnetic Resonance Imaging (fMRI)}

During fMRI scanning, each participant was exposed to six individually tailored audiotaped scripts (two neutralrelaxing, two stressful and two appetitive), which were presented using headphones in a randomized, counterbalanced order. Each script was $2.5 \mathrm{~min}$ in length and was preceded by a $1.5-\mathrm{min}$ baseline period and followed by a 1min quiet recovery period. During the baseline and recovery periods, participants lay quietly in the scanner. Immediately before and immediately following each trial, participants provided ratings of their subjective anxiety ('tense, anxious and/or jittery') and food craving ('imagine you have [subject's favorite food] in front of you right now, how much do you want [subject's favorite food] right now?') on a scale ranging from 0 ('not at all') to 10 ('more than ever'). These rating scales were presented verbally by the member of the research team overseeing the scanning session. Participants responded verbally, and the researcher recorded their responses. Immediately before the start of the scanning session and immediately subsequent to its conclusion, participants also provided hunger ratings on a scale ranging from 0 to 10 (also done verbally, researcher documented responses). Average ratings following each trial type for PCE and NCE adolescents are shown in Supplementary Figures S2 and S3.

In order to prevent any influence of emotion induced during the preceding trial, each trial was followed by a 2 -min progressive relaxation session and the next trial did not commence until anxiety and craving ratings returned to baseline. In order to confirm increases in anxiety and craving ratings following exposure to stressful and favoritefood cues, respectively, subjective ratings taken before and subsequent to cue exposure were compared using paired-sample $t$-tests. For further details on script generation or within scanner presentation, see Sinha (2009), Hommer et al (2012), and Jastreboff et al (2013).

\section{Image Acquisition}

All images were acquired using a $3 \mathrm{~T}$ Trio MRI and the same functional and structural acquisition parameters, as have been reported in previous publications using this task (Sinha et al, 2005; Jastreboff et al, 2011; Seo et al, 2011; Hommer et al, 2012; Potenza et al, 2012; Jastreboff et al, 2013). Anatomical images of the functional slice locations were acquired using a spin-echo sequence in the axial plane parallel to the anterior-posterior commissure line (repetition time/echo time $(\mathrm{TR} / \mathrm{TE})=300 / 2.46 \mathrm{~ms}$, bandwidth $=310 \mathrm{~Hz} /$ pixel, flip angle $=60^{\circ}$, field of view $(F O V)=220 \mathrm{~mm} \times 220$ $\mathrm{mm}$, matrix $=256 \times 256$, slice thickness $=4 \mathrm{~mm}$ (no skip), 32 slices). The purpose of this spin-echo sequence in the axial plane (2D anatomical) is to use a higher in-plane resolution $\left(256 \times 256 \mathrm{~mm}^{2}\right)$ instead of the functional resolution $(64 \times$ $64 \mathrm{~mm}^{2}$ ) for registration to the individual 3D structural image (see registration details below).

Functional data were acquired using a single-shot gradient echo-planar image (EPI) sequence $(\mathrm{TR} / \mathrm{TE}=$ $2000 / 25 \mathrm{~ms}$, flip angle $=85^{\circ}, \quad F O V=220 \mathrm{~mm} \times 220 \mathrm{~mm}$, matrix $=64 \times 64$, slice thickness $=4 \mathrm{~mm}$ (no skip), 32 slices). Structural data were acquired using a sagittal highresolution T1-weighted 3D magnetization-prepared-rapidgradient-echo (MPRAGE) sequence $(\mathrm{TR} / \mathrm{TE}=2530 / 3.34$ / $2.77 \mathrm{~ms}$, flip angle $=7^{\circ}, \mathrm{FOV}=256 \mathrm{~mm} \times 256 \mathrm{~mm}$, matrix $=$ $256 \times 256,176$ slices, $1 \mathrm{~mm}^{3}$ isotropic voxels).

\section{Image Analysis}

Functional imaging analyses were conducted with the BioImage Suite (www.bioimagesuite.org) using the same analysis approach as has been reported in previous studies using the guided-imagery paradigm (eg, Sinha et al, 2005; Jastreboff et al, 2011; Seo et al, 2011; Hommer et al, 2012; Potenza et al, 2012; Jastreboff et al, 2013). The BioImage Suite uses motion-correction algorithms from SPM5 (Wellcome Functional Imaging Laboratory, London, UK). 


\section{PreProcessing and First-Level Analyses}

Data were motion corrected using SPM5 (Wellcome Functional Imaging Laboratory), and trials with linear motion in excess of $1.5 \mathrm{~mm}$ or with rotation $>2^{\circ}$ were excluded. All subsequent analytic steps were conducted using algorithms developed in-house for the BioImage Suite as in our previous publications using this task (eg, Sinha et al, 2005; Jastreboff et al, 2011; Seo et al, 2011; Hommer et al, 2012; Potenza et al, 2012; Jastreboff et al, 2013). Individual participant data were analyzed using general linear models (GLMs) for each voxel, including time during imagery as a regressor $(2.5 \mathrm{~min})$ as compared with the baseline resting period $(1.5 \mathrm{~min})$ for each trial per condition. These images were then temporally filtered by including drift correction in the GLM. Drift regressors were used to remove the mean time course, linear trend, quadratic trend, and cubic trend for each run. The recovery period (1 min) was excluded from the data analysis to prevent carryover effects from the imagery period. Each trial was normalized against the immediate baseline period preceding the script and then two trials of the same type were averaged. Functional images were spatially smoothed with a 6-mm Gaussian kernel, resulting in normalized betamaps in the acquired space $(3.44 \mathrm{~mm} \times 3.44 \mathrm{~mm} \times 4 \mathrm{~mm})$.

Registration proceeded after three computations. First, a linear registration was computed from the individual functional image to the corresponding individual participant 2D anatomical image. Second, a linear registration was computed from the $2 \mathrm{D}$ anatomical image to the individual participants' 3D structural image. Finally, a non-linear registration was computed between the individual 3D image and a standard reference 3D image (the Colin27 Brain; (Holmes et al, 1998)). All three registrations were concatenated and applied as one registration to the normalized beta-maps in order to bring the data to a common reference space.

\section{Second-Level Analyses}

Group-level two-tailed $t$-tests were conducted using the BioImage Suite in order to identify any between-group differences (PCE/NCE) in BOLD-signal responses during the each of the three conditions (neutral-relaxing, stress, favoritefood). Family-wise-error (FWE) correction for multiple comparisons was conducted using a Monte Carlo simulation with AlphaSim in AFNI. The AlphaSim parameters used were a smoothing kernel of $6 \mathrm{~mm}$, a connection radius of $6.296 \mathrm{~mm}$, and a resolution of $3.44 \mathrm{~mm} \times 3.44 \mathrm{~mm} \times 4 \mathrm{~mm}$ voxels. With these parameters, it was determined that an activation volume of 132 original voxels $\left(6248 \mathrm{~mm}^{3}\right)$ satisfied the $p<0.05$ threshold. Additionally, whole-brain correlational analyses were conducted using the BioImage Suite and were FWE-corrected for multiple comparisons in order to explore the relationship between brain activations and subjective ratings of anxiety and craving.

\section{RESULTS}

\section{Neural Responses to Stressful and Appetitive Cues}

During the favorite-food condition, as compared with the neutral-relaxing condition, NCE adolescents had decreased activity within the ACC accompanied by an increased activity within the visual cortex. During the stressful condition, as compared with the neutral-relaxing condition, NCE adolescents had decreased activity within the bilateral insula and ACC. Further details of findings from withingroup comparisons of neural activations during the stressful and favorite-food conditions ( $v s$ the neutral-relaxing condition) among NCE adolescents are shown in Supplementary Table S1 and Supplementary Figures S4 and S5.

During the favorite-food condition, as compared with the neutral-relaxing condition, PCE adolescents had decreased activity within the right cerebellum and visual cortex. During the stressful condition, as compared with the neutral-relaxing condition, PCE adolescents had increased activity within regions, including the left insula, putamen, inferior frontal gyrus, and right cerebellum, accompanied by decreased activity within regions, including the visual cortex, parahippocampal, and fusiform gyri. Further details of the findings from within-group comparisons of neural activations during the stressful and favorite-food conditions ( $v s$ the neutral-relaxing condition) among PCE adolescents are shown in Supplementary Table S2 and Supplementary Figures S6 and S7.

\section{Between-Group Differences}

Findings from whole-brain between-group comparisons of brain activations during the appetitive, stressful, and neutral/relaxing conditions are shown in Table 2 and Figure 1. During the favorite-food condition, PCE $v s$ NCE adolescents had decreased activity within the ACC, medial PFC, dorsolateral PFC (DLPFC), left caudate, left putamen, and left hypothalamus. During the neutral-relaxing condition, PCE vs NCE adolescents had increased activity within a cluster encompassing bilateral primary and association visual areas and decreased activity within clusters, including the middle and superior temporal gyri and angular and supramarginal gyri.

\section{Correlations between Neural Responses and Subjective Ratings}

Self-report ratings of hunger collected before and subsequent to scanning did not differ between PCE and NCE adolescents ( $p$ 's $>0.05$ ). Self-report ratings of subjective anxiety and craving ratings collected before and after exposure to each cue condition revealed increases in anxiety following exposure to both stressful $(t=2.43, p=0.02$; preimagery $($ mean $\pm S E)=3.59 \pm 0.39$; postimagery $=3.90 \pm$ $0.42)$ and favorite-food $(t=3.10, p=0.003$; preimagery $=3.30 \pm 0.38$; postimagery $=3.78 \pm 0.40)$ cues. Craving ratings increased following exposure to favorite-food $(t=5.16, p<0.001$; preimagery $=4.62 \pm 0.45$; postimagery $=$ $5.80 \pm 0.46)$ cues and decreased following exposure to stressful cues $(t=-2.54, p=0.02$; preimagery $=5.09 \pm 0.46$; postimagery $=4.73 \pm 0.49)$. Neither anxiety nor craving ratings were significantly altered from baseline following exposure to neutral-relaxing cues ( $t$ 's $<0.91$, $p$ 's $>0.37)$.

Whole-brain correlational analyses revealed associations between subjective anxiety and craving ratings and brain activations across the different cue conditions at 
Table 2 Group Differences During Personalized Appetitive, Stressful, and Neutral-Relaxing Scripts (Whole-Brain, Family-Wise-Error (FWE) Corrected $p<0.05$ )

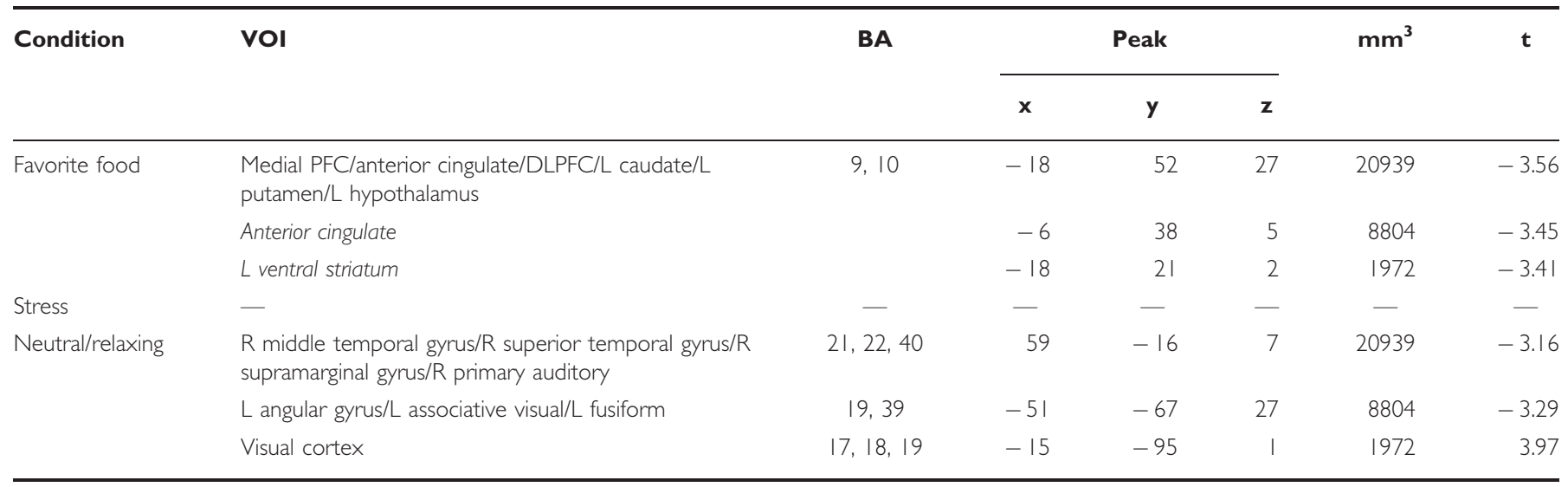

Abbreviatons: BA, Brodmann area; DLPFC, dorsolateral prefrontal cortex; L, left; R, right; VOI, volume of interest.

A negative $t$-value indicates PCE $<$ NCE, whereas a positive $t$-value indicates PCE $>$ NCE. Given the size of the clusters surviving whole-brain FWE correction for the favorite-food condition, $t$-values are also shown for peak subclusters (indicated in italics).

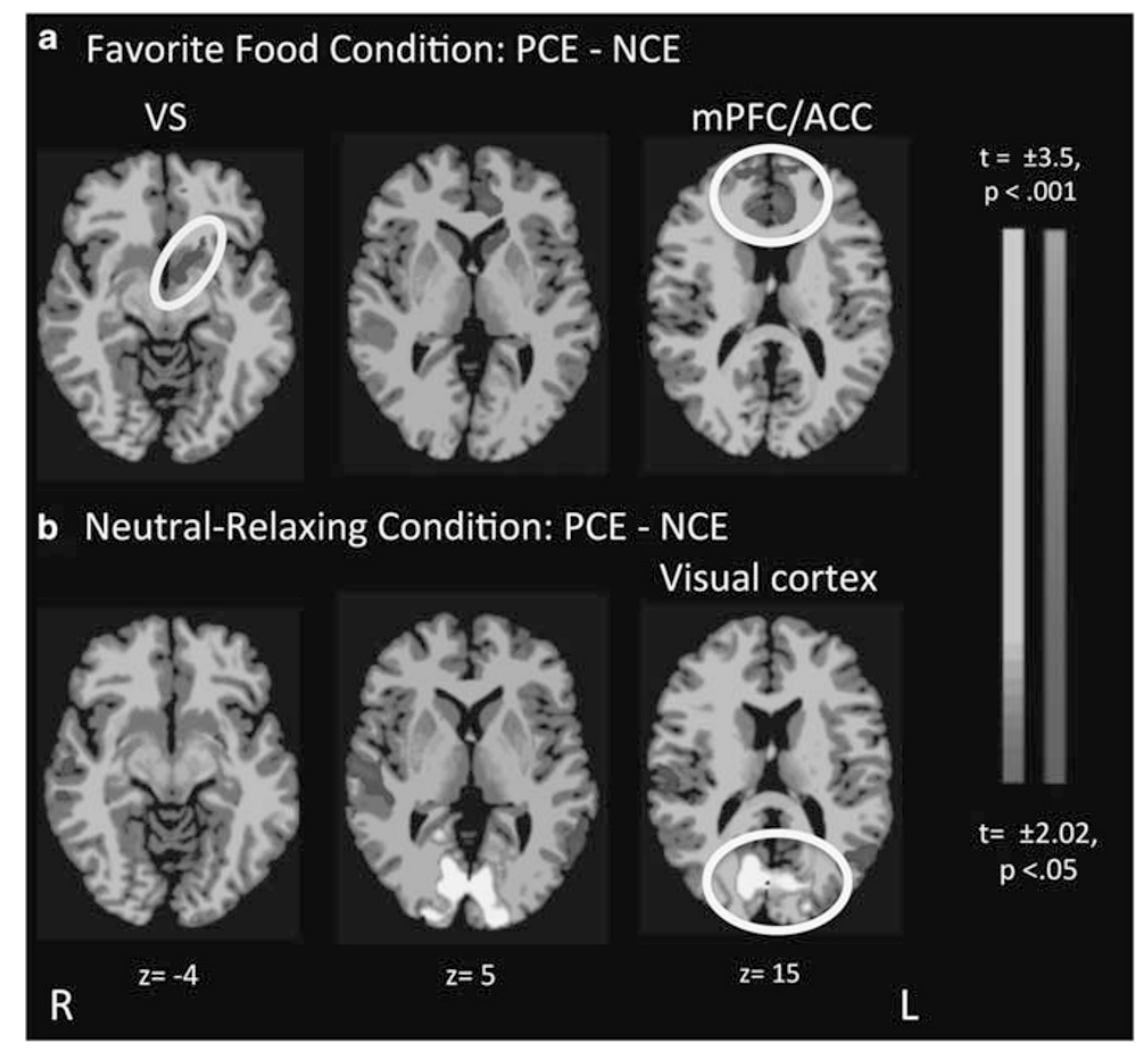

Figure I Whole-brain between-group differences during personalized favorite food (a) and neutral/relaxing (b) scripts (whole-brain, family-wise-error (FWE) corrected $p<0.05$ ). PCE, prenatal cocaine exposure; NCE, no prenatal cocaine exposure; VS, ventral striatum; mPFC, medial prefrontal cortex; ACC, anterior cingulate; R, right; L, left. Selected brain regions highlighted for emphasis using red, yellow, and white ovals. For further details on regions not highlighted above, please see Table 2. A full color version of this figure is available at Neuropsychopharmacology online.

pFWE $<0.01$ (see Table 3 and Figure 2; selected correlations shown in scatterplots in Supplementary Figures S8-S14).

\section{Subjective Anxiety}

There were no significant differences in subjective anxiety ratings (postexposure minus baseline) between PCE $v s$ NCE adolescents during any of the three imagery conditions ( $p$ 's $>0.32$ ). During the favorite-food condition, subjective anxiety ratings correlated negatively with activity within the left associative visual cortex among PCE adolescents (Figure 2). No associations between subjective anxiety ratings and brain activations during the favorite-food condition were observed among NCE adolescents. 
Table 3 Voxel-Wise, Whole-Brain Correlations Between Anxiety and Craving Ratings and Brain Activations During Personalized Appetitive, Stressful, and Neutral-Relaxing Scripts Among PCE adolescents (Whole-Brain, Family-Wise-Error (FWE) Corrected p<0.0 I)

\begin{tabular}{|c|c|c|c|c|c|c|}
\hline \multirow[t]{2}{*}{ vol } & \multirow[t]{2}{*}{ BA } & \multicolumn{3}{|c|}{ Peak } & \multirow[t]{2}{*}{$\mathrm{mm}^{3}$} & \multirow[t]{2}{*}{$\mathbf{r}$} \\
\hline & & $\mathbf{x}$ & $y$ & z & & \\
\hline \multicolumn{7}{|l|}{ PCE } \\
\hline$L$ associative visual cortex & 19 & -12 & -85 & 31 & 2579 & -0.73 \\
\hline \multicolumn{7}{|l|}{ PCE } \\
\hline Medial inferior anterior cerebellum & & -8 & -50 & -35 & 4570 & -0.76 \\
\hline Medial superior posterior cerebellum & & 36 & -60 & -40 & 11425 & -0.80 \\
\hline Brainstem $/ L$ fusiform gyrus & & -17 & -34 & -8 & 6492 & -0.78 \\
\hline $\mathrm{R}$ OFC/R IFG & 11,47 & 37 & 22 & -9 & $|53|$ & -0.68 \\
\hline
\end{tabular}

Anxiety during neutral/relaxing condition

PCE

$L$ inferior temporal gyrus/ $L$ middle temporal gyrus

$\begin{array}{crrrrr}20,21 & -45 & -34 & -9 & 4957 & -0.71 \\ 19 & 46 & -72 & -8 & 8998 & -0.80 \\ 10 & -6 & 46 & 7 & 2111 & -0.64 \\ 39 & -36 & -58 & 24 & 2630 & -0.78 \\ 8,9 & -21 & 39 & 33 & 3564 & -0.73 \\ 8,9 & -5 & 14 & 64 & 14277 & -0.79 \\ 6,8,9 & -32 & 9 & 34 & 4904 & -0.74 \\ & 6 & -5 & 34 & 2720 & -0.68 \\ 7 & -11 & -45 & 34 & 11819 & -0.76 \\ 39,40,47 & 39 & -45 & 32 & 7704 & -0.76 \\ & 24 & -12 & 42 & 1859 & -0.67\end{array}$

R fusiform gyrus/R parahippocampal gyrus/R hippocampus

Anterior cingulate

$L$ angular gryrus

Medial PFC

R DLPFC

L DLPFC

Anterior cingulate

Posterior cingulate

$\mathrm{R}$ angular gyrus/R supramarginal gyrus/R IFG/R primary sensory

cortex/R primary motor cortex

Premotor cortex (peak in white matter next to BA 6)

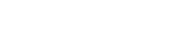

Food craving during favorite-food condition

PCE

L DLPFC/L anterior PFC

9,10

$-21$

43

25

|79|

$-0.73$

Abbreviations: BA, Brodmann area; DLPFC, dorsolateral prefrontal cortex; IFG, inferior frontal gyrus; L, left; OFC, orbitofrontal cortex; PFC, prefrontal cortex; R, right; VOI, volume of interest.

Anxiety ratings correlated negatively with brain activity during the stressful script within the cerebellum, brainstem, posterior cingulate, premotor area, right orbitofrontal cortex (OFC), right inferior frontal gyrus (IFG), right pars opercularis, and left fusiform gyrus among PCE adolescents (Figure 2). There were no significant associations between brain activations during the stressful condition and subjective anxiety ratings among NCE adolescents.

Subjective anxiety ratings correlated negatively with brain activity during the neutral-relaxing condition within the anterior and posterior cingulate, medial PFC, DLPFC, angular gyrus, left inferior temporal gyrus, left middle temporal gyrus, right fusiform gyrus, right parahippocampal gyrus, right hippocampus, right supramarginal gyrus, right inferior frontal gyrus, right primary sensory cortex, right primary motor cortex, and right premotor cortex among PCE adolescents (Figure 2). No significant associations between subjective anxiety ratings and brain activations during the neutral-relaxing condition were observed among NCE adolescents.

\section{Food Craving}

There were no significant differences in food-craving ratings (postexposure minus baseline) between PCE $v s$ NCE adolescents for any of the three cue conditions ( $p$ 's $>0.46$ ). Food-craving ratings correlated negatively with brain activations during the favorite-food condition within the left DLPFC and anterior PFC among PCE adolescents (Figure 2). No significant associations between food-craving ratings 


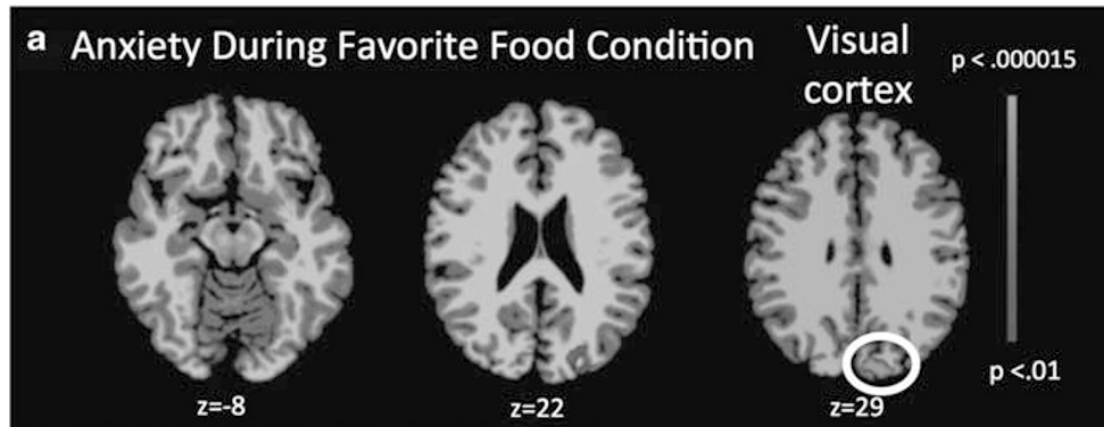

b Anxiety During Stress Condition
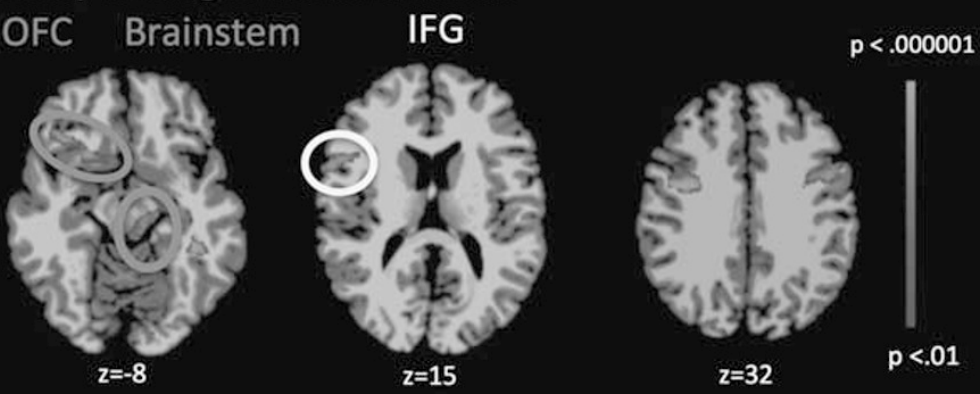
c Anxiety During Neutral/Relaxing Condition Para/Hippocampus ACC/mPFC
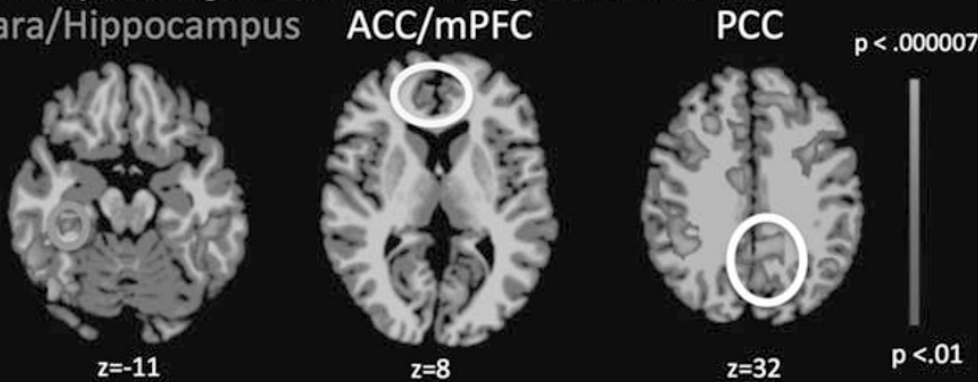

d Food Craving During Favorite Food Condition

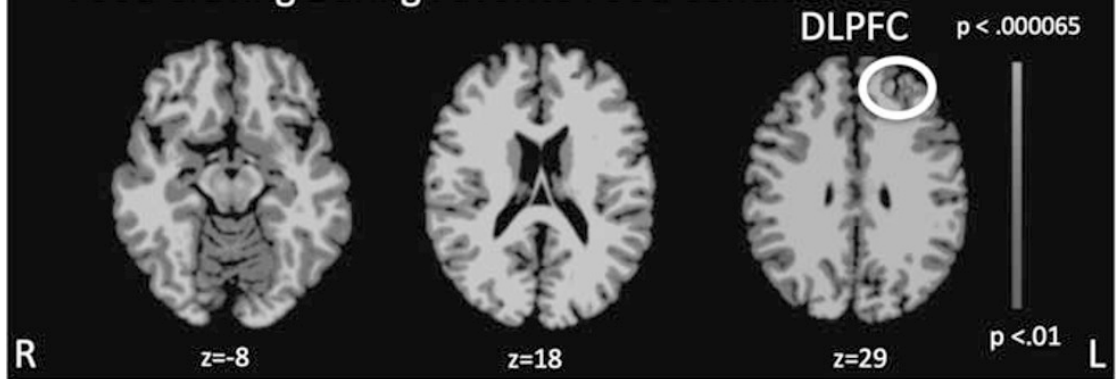

Figure 2 Voxel-wise, whole-brain correlations between anxiety and craving ratings and brain activations during personalized appetitive, stressful, and neutral/relaxing scripts among PCE adolescents (whole-brain, family-wise-error (FWE) corrected $p<0.0$ ). Panel (a) shows correlations between anxiety and neural responses during the favorite food condition. Panel (b) shows correlations between anxiety and neural responses during the stress condition. Panel (c) shows correlations between anxiety and neural responses during the neutral/relaxing condition. Panel (d) shows correlations between food craving and neural responses during the favorite food condition. PCE, prenatal cocaine exposure; NCE, no prenatal cocaine exposure; ACC, anterior cingulate; PCC, posterior cingulate; mPFC, medial prefrontal cortex; DLPFC, dorsolateral prefrontal cortex; OFC, orbitofrontal cortex; IFG, inferior frontal gyrus; $\mathrm{R}$, right; L, left. Selected brain regions highlighted for emphasis using red, yellow, and white ovals. For scatterplots showing correlations between BOLD responses in the highlighted regions and subjective ratings among PCE and NCE adolescents, respectively, please refer to Supplementary Materials. For further details on regions not highlighted above, please see Table 3. A full color version of this figure is available at Neuropsychopharmacology online.

and brain activations during the favorite-food condition were observed among NCE adolescents. There were no significant associations between food-craving ratings and brain activations during the stressful or neutral-relaxing conditions among PCE or NCE adolescents.

\section{DISCUSSION}

Partially consistent with our first hypothesis, PCE adolescents demonstrated relatively blunted activations within corticostriatal-limbic structures during exposure to 
appetitive cues but did not differ from NCE adolescents during exposure to stressful cues. Our findings are consistent with those from previous studies conducted in adolescent populations, suggesting that reduced VS activation may be a vulnerability marker for the development of addictive disorders (Peters et al, 2011). Notably, our findings differ from the studies of adults with cocaine dependence (Potenza et al, 2012), suggesting that prenatal exposure to cocaine might differentially impact neural responses to stress and food cues, possibly related to cocaine effects on the development of cortico-striatal-limbic pathways serving the processing of emotions and rewards.

Contrary to our second hypothesis, subjective anxiety and craving ratings were largely negatively correlated with brain activations among PCE adolescents. Specifically, among PCE adolescents, neural responses to neutral-relaxing, stressful, and appetitive cues were generally inversely associated with subjective anxiety and craving ratings; however, this pattern was not seen among NCE adolescents, despite similar anxiety ratings across both groups and strict exclusion criteria for potential artifacts such as motion across both participant groups (see Supplementary Materials for details). Taken together, these findings suggest alterations among adolescents with PCE in the relationships between subjective responses to stressful and appetitive cues and regional brain activations in areas implicated in emotion regulation (Nikolova et al, 2012) and motivational control (Sinha, 2001; Sinha and Li, 2007; Sinha, 2009). These findings suggest a possible neurobiological mechanism for behavioral problems observed among children with PCE (Mayes, 2002; Buckingham-Howes et al, 2013), as well as for increased rates of weight problems and substanceuse behaviors among adolescents with PCE (Delaney-Black et al, 2011; Rando et al, 2013; Richardson et al, 2013).

\section{Neural Responses to Favorite-Food Cues}

During the favorite-food condition, PCE $v s$ NCE adolescents demonstrated less brain activity within regions, including the DLPFC, ACC, hypothalamus, and VS The VS is involved in reward processing, including aspects related to emotional and motivational control and the encoding of both rewarding and aversive stimuli. Previous studies suggest that diminished VS activity may be a vulnerability marker for the development of future substance-use behaviors among adolescents (Peters et al, 2011). Thus the diminished VS activity observed in this study may be linked to an increased vulnerability to the development of addictive disorders among adolescents with PCE (Peters et al, 2011). This interpretation is consistent with research suggesting increased rates of substance use among adolescents with PCE (Delaney-Black et al, 2011; Rando et al, 2013; Richardson et al, 2013); however, further research is needed to examine more directly associations between altered VS responses and rates of substance use during adolescence in this and other similar cohorts.

The diminished activity observed among PCE adolescents within the hypothalamus, VS, and regions of the PFC may relate to vulnerabilities for obesity: reduced BOLD responses within these and other regions have been reported among adults with obesity and binge-eating disorder (Balodis et al, 2013a, b) and longitudinal data suggest increased rates of obesity among children with PCE
(LaGasse et al, 2011). Furthermore, diminished activation of cortico-striatal regions during reward processing has been linked to persistent bingeing in binge-eating disorder, suggesting that underactivation relates importantly to behaviors relating to poor weight control (Balodis et al, 2013a, b). The PCE and NCE adolescents included in this study were matched well for BMI; thus it is also possible that the blunted activations observed among PCE adolescents may relate to future propensities for weight gain, and this possibility warrants direct investigation. Additionally, the blunted activations in PCE adolescents might relate to a general downregulation of reward-related neurocircuitry, and future studies should thus examine propensities for PCE adolescents to develop problems in a range of rewardrelated behaviors.

Adolescents with PCE also had reduced activity within a cluster encompassing the dorsomedial PFC and ACC during the favorite-food condition. This region has been previously implicated in cocaine craving and dependence (Wexler et al, 2001) and is involved in inhibitory 'top-down' emotional and cognitive-control processes (Etkin et al, 2006; Chiew and Braver, 2011; Giuliani et al, 2011). Cognitivecontrol mechanisms naturally develop with age in typical development (Crone et al, 2004), and may be particularly relevant for the generation of appropriate coping strategies in response to substance-using situations (Rando et al, 2013). Thus these findings suggest that PCE's teratogenic effects may include functional alterations to regions involved in the maintenance of craving states and cognitive-control processes previously implicated in obesity and addictive disorders (Potenza et al, 2012; Balodis et al, 2013a, b). The extent to which these alterations in ACC activity may represent a vulnerability marker for the development of subsequent harmful substance-use behaviors remains to be determined.

The DLPFC is implicated in a range of processes relevant to the development of addictions, including motivational, inhibitory, and attentional control and decision-making processes (Goldstein and Volkow, 2011). Thus, it is possible that the decreased PFC activity observed among PCE adolescents may relate to decreased cognitive control during craving states (Kober et al, 2010), although this possibility warrants direct examination. This latter interpretation is strengthened by the negative association between self-reported craving and DLPFC activity during exposure to favorite-food cues observed among PCE adolescents. Moreover, during both neutral-relaxing and stress conditions, PCE adolescents reported increased craving (relative to NCE adolescents), suggesting that alterations in reward/craving mechanisms may also be altered across different emotional states, as has been reported in adult studies of cocaine dependence (Potenza et al, 2012).

\section{Neural Responses to Neutral-Relaxing Cues}

In comparison with NCE adolescents, PCE adolescents had increased activity within the primary visual cortex during the neutral-relaxing condition. In addition to its role in visual perception, the visual cortex is also involved in mental imagery formation (Ganis et al, 2004). Thus the heightened activation within the visual cortex might relate to increased engagement in mental imagery during the 
neutral-relaxing condition among PCE adolescents. Conversely, heightened visual cortex activation might relate to a decreased ability to engage in neutral imagery (leading to increased activity via increased cognitive load). Further research is needed to explore these other hypotheses. Mental imagery may contribute to emotional processing and has been examined in the development of novel psychotherapies (Heyes et al, 2013). As such, further research into the relative impact of PCE on mental imagery formation might be efficacious in guiding treatment efforts aimed at addressing behavioral problems in this and other at-risk populations.

\section{Correlations between Anxiety and Brain Activations}

Across both NCE and PCE adolescents, anxiety ratings increased following exposure to favorite-food cues. This is consistent with our previous findings of increased anxiety ratings following exposure to drug-related cues among adults with cocaine dependence (Potenza et al, 2012) and suggests that favorite-food cues may be an effective analogue for studying drug-like craving responses in nonaddicted populations.

Among PCE adolescents, increased anxiety ratings were associated with decreased activity in response to stress cues within regions involved in the processing of stressful and threatening stimuli (eg, brainstem) (Liddell et al, 2005) as well as in regions implicated in cognitive control processes, including aspects related to motivational control, decision-making, and behavioral regulation (eg, IFG, OFC) (Goldstein and Volkow, 2011). Similarly, during the neutralrelaxing condition, increased anxiety ratings were associated with decreased activity within the hippocampus, ACC, and DLPFC among PCE adolescents. Notably, all of these regions have been previously implicated in cue-induced cocaine craving in studies of adult cocaine users (Garavan, 2000; Bonson et al, 2002; Goldstein et al, 2007; Potenza et al, 2012). Overall, these findings suggest that PCE may lead to alterations in frontal-limbic circuitry involved in stress processing and cognitive control that are related to individual differences in subjective anxiety during adolescence.

\section{Correlations between Food Craving and Brain Activations}

Among PCE adolescents, increased food-craving ratings were associated with decreased engagement of the DLPFC during the favorite-food condition. Previous research has demonstrated a positive association between successful downregulation of subjective craving states and increases in DLPFC activity in response to both substance and foodrelated cues (Kober et al, 2010). Thus the negative association between DLPFC activity and craving observed among PCE adolescents in this study may represent a vulnerability marker for the development of future substance-use behaviors and/or diminished control over eating behaviors.

\section{Developmental Implications}

Adolescence is a significant epoch for the neurodevelopment of key structures involved in emotional processing and motivational control-notably, regions of the PFC involved in inhibitory control processes (Chambers and Potenza, 2003). Preclinical data additionally suggest that maturation of striatum and other regions receiving strong dopaminergic innervations continues throughout adolescence (Galvan, 2010). In this study, we observed decreased activity in both the PFC and striatum among adolescents with PCE, which, as discussed, may relate to the development of substance- and eating-related disorders. However, these structures are involved in multiple processes (and the reduced responses observed were not constrained to only these regions); thus the larger developmental implications of these findings remains to be determined. For example, given the role of the striatum in habit formation and learning, these findings might relate to the working memory deficits (Mayes et al, 2007) reported among children with PCE. Similarly, reductions in PFC activations may relate to previously reported impairments in response inhibition (Accornero et al, 2007), which could influence multiple developmental processes (such as the appropriate inhibition of negative or anxiety-provoking thoughts or actions) other than substance-use initiation.

\section{Strengths and Limitations}

This study has several strengths, including its selection of a group of adolescents well matched for age, gender, and BMI with and without PCE who have been followed longitudinally since birth and the use of a well-validated, personalized imagery task. This study is limited by a relatively small sample of largely disadvantaged youths (Mayes et al, 2005; Hommer et al, 2012). Future studies should investigate older PCE and NCE individuals and investigate the influence of substance-use disorders on brain function within these groups. This study did not systematically assess for current education level. However, all of the adolescents included in this study were currently enrolled in school, and PCE and NCE adolescents groups did not differ in age or IQ, suggesting that it is likely that the two groups were comparable with respect to education level. Although the NCE and PCE adolescent groups were well matched for age and gender, this study did not include a formal assessment of pubertal stage, and future studies should aim to specifically match on this variable.

\section{Conclusions and Future Directions}

Overall, our findings suggest functional brain alterations among individuals with PCE within regions implicated in emotional regulation, motivational control, and addiction vulnerability during adolescence. These findings suggest similarities with those from studies of chronic cocaine use in adults (Sinha et al, 2005; Potenza et al, 2012) and suggest significant effects of PCE on adolescent neural functioning that warrant further investigation in larger sample sizes, possibly including adolescents with and without problematic substance-use behaviors. Finally, the relationship between gender and PCE remains underresearched. Given the elevated rates of eating-disorderrelated pathology among adolescent girls (in comparison with boys) (Merikangas et al, 2010), exploring how gender might interact with in utero cocaine exposure in relation to 
eating-related pathology will be an important step for future investigations.

\section{FUNDING AND DISCLOSURE}

MNP has received financial support or compensation for the following: MNP has consulted for and advised Boehringer Ingelheim, Lundbeck, and Ironwood; has consulted for and has financial interests in Somaxon; has received research support from the National Institutes of Health, Veteran's Administration, Mohegan Sun Casino, the National Center for Responsible Gaming and its affiliated Institute for Research on Gambling Disorders, and Forest Laboratories, Ortho-McNeil, Oy-Control/Biotie, GlaxoSmithKline, and Psyadon Pharmaceuticals; has participated in surveys, mailings, or telephone consultations related to drug addiction, impulse control disorders, or other health topics; has consulted for gambling entities, law offices, and the federal public defender's office in issues related to impulse control disorders; provides clinical care in the Connecticut Department of Mental Health and Addiction Services Problem Gambling Services Program; has performed grant reviews for the National Institutes of Health and other agencies; has guest-edited journal sections; has given academic lectures in grand rounds, CME events, and other clinical or scientific venues; and has generated books or book chapters for publishers of mental health texts. The other authors declare no conflict of interest.

\section{ACKNOWLEDGEMENTS}

The study was supported by the National Institutes of Health; Contract grant numbers: P50 DA016556, P50 DA09241, UL1-DE19586, RL1 AA017539, R01 DA006025, R01 DA017863, K05 DA020091; Contract grant sponsor: the Office of Research on Women's Health, the NIH Roadmap for Medical Research/Common Fund. SWY receives salary support from a NIDA-funded T32 Postdoctoral Fellowship Program (DA007238-23, PI: Petrakis).

\section{REFERENCES}

Accornero V, Accornero A, Amado C, Morrow L, Xue J, Anthony E et al (2007). Impact of prenatal cocaine exposure on attention and response inhibition as assessed by continuous performance tests. J Dev Behav Pediatr 28: 195-205.

Ackerman JP, Riggins T, Black MM (2010). A review of the effects of prenatal cocaine exposure among school-aged children. Pediatrics 125: 554-565.

Andersen SL, Teicher MH (2008). Stress, sensitive periods and maturational events in adolescent depression. Trends Neurosci 31: 183-191.

Balodis IM, Grilo CM, Kober H, Worhunsky PD, White MA, Stevens MC et al (2013a). A pilot study linking reduced fronto-Striatal recruitment during reward processing to persistent bingeing following treatment for binge-eating disorder. Int $J$ Eat Disord Early Online 47: 376-384.

Balodis IM, Kober H, Worhunsky PD, White MA, Stevens MC, Pearlson GD et al (2013b). Monetary reward processing in obese individuals with and without binge eating disorder. Biol Psychiatry 73: 877-886.
Bonson K, Grant SJ, Contoreggi CS, Links JM, Metcalfe J, Weyl L et al (2002). Neural systems and cue-induced cocaine craving. Neuropsychopharmacology 26: 376-386.

Bridgett DJ, Mayes LC (2011). Development of inhibitory control among prenatally cocaine exposed and non-cocaine exposed youths from late childhood to early adolescence: the effects of gender and risk and subsequent aggressive behavior. Neurotoxicol Teratol 33: 47-60.

Buckingham-Howes S, Berger SS, Scaletti LA, Black MM (2013). Systematic review of prenatal cocaine exposure and adolescent development. Pediatrics 131: e1917-e1936.

Chambers RA, Potenza MN (2003). Neurodevelopment, impulsivity and adolescent gambling. J Gambl Stud 19: 53-84.

Chiew KS, Braver TS (2011). Neural circuitry of emotional and cognitive conflict revealed through facial expressions. PLoS One 6: e17635.

Cohen S, Kamarck T, Mermelstein R (1983). A global measure of perceived stress. J Health Soc Behav 24: 385-396.

Crone EA, Richard Ridderinkhof K, Worm M, Somsen RJM, Van Der Molen MW (2004). Switching between spatial stimulusresponse mappings: a developmental study of cognitive flexibility. Dev Sci 7: 443-455.

Delaney-Black V, Chiodo LM, Hannigan JH, Greenwald MK, Janisse J, Patterson G et al (2011). Prenatal and postnatal cocaine exposure predict teen cocaine use. Neurotoxicol Teratol 33: 110-119.

Eshel N, Nelson EE, Blair RJ, Pine DS, Ernst M (2007). Neural substrates of choice selection in adults and adolescents: development of the ventrolateral prefrontal and anterior cingulate cortices. Neuropsychologia 45: 1270-1279.

Etkin A, Egner T, Peraza DM, Kandel ER, Hirsch J (2006). Resolving emotional conflict: a role for the rostral anterior cingulate cortex in modulating activity in the amygdala. Neuron 51: $871-882$.

Galvan A (2010). Adolescent development of the reward system. Front Hum Neurosci 4: 6.

Ganis G, Thompson WL, Kosslyn SM (2004). Brain areas underlying visual mental imagery and visual perception: an fMRI study. Cogn Brain Res 20: 226-241.

Garavan H, Pankiewicz J, Bloom A, Cho J-K, Sperry L, Ross TJ et al (2000). Cue-induced cocaine craving: neuroanatomical specificity for drug users and drug stimuli. Am J Psychiatry 157: 1789-1798.

Giuliani NR, Drabant EM, Gross JJ (2011). Anterior cingulate cortex volume and emotion regulation: is bigger better? Biol Psychol 86: 379-382.

Goldstein RZ, Tomasi D, Rajaram S, Cottone LA, Zhang L, Maloney $\mathrm{T}$ et al (2007). Role of the anterior cingulate and medial orbitofrontal cortex in processing drug cues in cocaine addiction. Neurosci 144: 1153-1159.

Goldstein RZ, Volkow ND (2011). Dysfunction of the prefrontal cortex in addiction: neuroimaging findings and clinical implications. Nat Rev Neurosci 12: 652-669.

Harvey J, Romano A, Gabriel M, Simansky K, Du W, Aloyo V et al (2001). Effects of prenatal exposure to cocaine on the developing brain: anatomical, chemical, physiological and behavioral consequences. Neurotox Res 3: 117-143.

Harvey JA (2004). Cocaine effects on the developing brain: current status. Neurosci Biobehav Rev 27: 751-764.

Heyes S, Lau J, Holmes E (2013). Mental imagery, emotion and psychopathology across child and adolescent development. Dev Cogn Neurosci 5: 119-133.

Holmes CJ, Hoge R, Collins L, Woods R, Toga AW, Evans AC (1998). Enhancement of MR images using registration for signal averaging. J Comput Assist Tomogr 22: 324-333.

Hommer RE, Seo D, Lacadie CM, Chaplin TM, Mayes LC, Sinha R et al (2012). Neural correlates of stress and favorite-food cue exposure in adolescents: a functional magnetic resonance imaging study. Hum Brain Mapp 34: 2561-2573. 
Jastreboff AM, Potenza MN, Lacadie C, Hong KA, Sherwin RS, Sinha R (2011). Body mass index, metabolic factors, and striatal activation during stressful and neutral-relaxing states: an FMRI study. Neuropsychopharmacology 36: 627-637.

Jastreboff AM, Sinha R, Lacadie C, Small DM, Sherwin RS, Potenza MN (2013). Neural correlates of stress- and food cue-induced food craving in obesity: association with insulin levels. Diabetes Care 36: 394-402.

Kaufman AS (1983). In: Kaufman Assessment Battery for Children. Administration and Scoring Manual. American Guidance Service Inc., Circle Pines, MN, USA.

Kober H, Mende-Siedlecki P, Kross EF, Weber J, Mischel W, Hart CL et al (2010). Prefrontal-striatal pathway underlies cognitive regulation of craving. Proc Natl Acad Sci USA 107: 14811-14816.

LaGasse LL, Gaskins RB, Bada HS, Shankaran S, Liu J, Lester BM et al (2011). Prenatal cocaine exposure and childhood obesity at nine years. Neurotoxicol Teratol 33: 188-197.

Lang P, Kozak MJ, Miller GA, Levin DN, McLean A Jr (1980). Emotional imagery: conceptual structure and pattern of somatovisceral response. Psychophysiology 17: 179-192.

Li Z, Coles CD, Lynch ME, Hamann S, Peltier S, LaConte S et al (2009). Prenatal cocaine exposure alters emotional arousal regulation and its effects on working memory. Neurotoxicol Teratol 31: 342-348.

Li Z, Santhanam P, Coles CD, Lynch ME, Hamann S, Peltier S et al (2011). Increased 'default mode' activity in adolescents prenatally exposed to cocaine. Hum Brain Mapp 32: 759-770.

Liddell BJ, Brown KJ, Kemp AH, Barton MJ, Das P, Peduto A et al (2005). A direct brainstem-amygdala-cortical 'alarm' system for subliminal signals of fear. Neuroimage 24: 235-243.

Liu J, Lester BM (2011). Reconceptualizing in a dual-system model the effects of prenatal cocaine exposure on adolescent development: a short review. Int J Dev Neurosci 29: 803-809.

Mayes L, Molfese D, Key A, Hunter N (2005). Event-related potentials in cocaine-exposed children during a Stroop task. Neurotoxicol Teratol 27: 797-813.

Mayes L, Snyder PJ, Langlois E, Hunter N (2007). Visuospatial working memory in school-aged children exposed in utero to cocaine. Child Neuropsychol 13: 205-218.

Mayes LC (2002). A behavioral teratogenic model of the impact of prenatal cocaine exposure on arousal regulatory systems. Neurotoxicol Teratol 24: 385-395.

McCarthy DM, Bhide PG (2012). Prenatal cocaine exposure decreases parvalbumin-immunoreactive neurons and GABA-toprojection neuron ratio in the medial prefrontal cortex. Dev Neurosci 34: 174-183.

Merikangas KR, He J-p, Burstein M, Swanson SA, Avenevoli S, Cui L et al (2010). Lifetime prevalence of mental disorders in U.S. adolescents: results from the National Comorbidity Survey Replication-Adolescent Supplement (NCS-A). J Am Acad Child Adolesc Psychiatry 49: 980-989.

Nikolova YS, Bogdan R, Brigidi BD, Hariri AR (2012). Ventral striatum reactivity to reward and recent life stress interact to predict positive affect. Biol Psychiatry 72: 157-163.
Peters J, Bromberg U, Schneider S, Brassen S, Menz M, Banaschewski $\mathrm{T}$ et al (2011). Lower ventral striatal activation during reward anticipation in adolescent smokers. Am J Psychiatry 168: 540-549.

Potenza MN, Hong K-iA, Lacadie CM, Fulbright RK, Tuit KL, Sinha R (2012). Neural correlates of stress-induced and cueinduced drug craving: influences of sex and cocaine dependence. Am J Psychiatry 169: 406-414.

Rando K, Chaplin TM, Potenza MN, Mayes L, Sinha R (2013). Prenatal cocaine exposure and gray matter volume in adolescent boys and girls: relationship to substance use initiation. Biol Psychiatry 74: 482-489.

Richardson GA, Larkby C, Goldschmidt L, Day NL (2013). Adolescent initiation of drug use: effects of prenatal cocaine exposure. J Am Acad Child Adolesc Psychiatry 52: 37-46.

SAMHSA (2013). Results from the 2012 National Survey on Drug Use and Mental Health. Substance Abuse and Mental Health Services Administration: Rockville, MD, USA.

Seo D, Jia Z, Lacadie CM, Tsou KA, Bergquist K, Sinha R (2011). Sex differences in neural responses to stress and alcohol context cues. Hum Brain Mapp 32: 1998-2013.

Seo D, Tsou KA, Ansell EB, Potenza MN, Sinha R (2014). Cumulative adversity sensitizes neural response to acute stress: association with health symptoms. Neuropsychopharmacology 39: $670-680$.

Shaffer D, Fisher P, Lucas CP, Dulcan MK, Schwab-Stone ME (2000). NIMH Diagnostic Interview Schedule for Children Version IV (NIMH DISC-IV): description, differences from previous versions, and reliability of some common diagnoses. J Am Acad Child Adolesc Psychiatry 39: 28-38.

Sheinkopf SJ, Lester BM, Sanes JN, Eliassen JC, Hutchison ER, Seifer R et al (2009). Functional MRI and response inhibition in children exposed to cocaine in utero. Dev Neurosci 31: 159-166.

Sinha R (2001). How does stress increase risk of drug abuse and relapse? Psychopharmacol 158: 343-359.

Sinha R (2009). Modeling stress and drug craving in the laboratory: implications for addiction treatment development. Addict Biol 14: 84-98.

Sinha R, Lacadie C, Skudlarski P, Fulbright R, Kosten T, Rounsaville B et al (2005). Neural activity associated with stress-induced cocaine craving: an fMRI study. Psychopharmacol 183: $171-180$.

Sinha R, Li C-S (2007). Imaging stress and drug cue-induced craving: association to relapse and clinical implications. Drug Alcohol Rev 26: 25-31.

Wang W, Nitulescu I, Lewis JS, Lemos JC, Bamford IJ, Posielski NM et al (2013). Overinhibition of corticostriatal activity following prenatal cocaine exposure. Ann Neurol 73: 355-369.

Wexler B, Gottschalk CH, Fulbright RF, Prohovnik I, Lacadie CM, Rounsaville BJ et al (2001). fMRI of cocaine craving. Am J Psychiatry 158: 86-95.

Wills TA, Vaccaro D, McNamara G (1992). The role of life events, family support, and competence in adolescent substance use: a test of vulnerability and protective factors. Am J Community Psychol 20: 349-374.

Supplementary Information accompanies the paper on the Neuropsychopharmacology website (http://www.nature.com/npp) 